\title{
Self-organized criticality model for brain plasticity
}

\author{
Lucilla de Arcangelis, ${ }^{1}$ Carla Perrone-Capano ${ }^{2}$ and Hans J. Herrmann ${ }^{3}$ \\ 1 Dept. of Information Engineering and CNISM, \\ Second University of Naples, 81031 Aversa (CE), Italy \\ 2 Dept. of Biological Sciences, University of Naples "Federico II", 80134. \\ Naples, Italy and IGB "A.Buzzati Traverso", CNR, 80131 Naples, Italy. \\ 3 Institute for Computer Applications 1, University of Stuttgart, \\ Pfaffenwaldring 27, D-70569 Stuttgart, Germany
}

\begin{abstract}
Networks of living neurons exhibit an avalanche mode of activity, experimentally found in organotypic cultures. Here we present a model based on self-organized criticality and taking into account brain plasticity, which is able to reproduce the spectrum of electroencephalograms (EEG). The model consists in an electrical network with threshold firing and activity-dependent synapse strenghts. The system exhibits an avalanche activity power law distributed. The analysis of the power spectra of the electrical signal reproduces very robustly the power law behaviour with the exponent 0.8 , experimentally measured in EEG spectra. The same value of the exponent is found on small-world lattices and for leaky neurons, indicating that universality holds for a wide class of brain models.
\end{abstract}

PACS numbers: 05.65.+b, 05.45.Tp, 89.75.-k, 87.19.La

Cortical networks exhibit diverse patters of activity, including oscillations, synchrony and waves. During neuronal activity, each neuron can receive inputs by thousands of other neurons and, when it reaches a threshold, redistributes this integrated activity back to the neuronal network. Recently it has been shown that another mode of activity is neuronal avalanches, with a dynamics similar to self-organized criticality (SOC) 1, 2, 3, 4], observed in organotypic cultures from coronal slices of rat cortex 5] where neuronal avalanches are stable for many hours [6]. The term SOC usually refers to a mechanism of slow energy accumulation and fast energy redistribution driving the system toward a critical state, where the distribution of avalanche sizes is a power law obtained without fine tuning: no tunable parameter is present in the model. The simplicity of the mechanism at the basis of SOC has suggested that many physical and biological phenomena characterized by power laws in the size distribution, represent natural realizations of the SOC idea. For instance, SOC has been proposed to model earthquakes 7,8$]$, the evolution of biological systems [9], solar flare occurrence 10, fluctuations in confined plasma 11] snow avalanches 12] and rain fall [13].

In order to monitor neural activities, different time series are usually analysed through power spectra and generally power-law decay is observed. A large number of time series analyses have been performed on medical data that are directly or indirectly related to brain activity. Prominent examples are EEG data which are used by neurologists to discern sleep phases, diagnose epilepsy and other seizure disorders as well as brain damage and disease [14, 15]. However, the interpretation of physiological mechanisms at the basis of EEG measurements is still controversial. Another example of a physiological function which can be monitored by time series analysis is the human gait which is controlled by the brain [16]. For all these time series the power spectrum, i.e. the square of the amplitude of the Fourier transformation double logarithmically plotted against frequency, generally features a power law at least over one or two orders of magnitude with exponents between 1 and 0.7. Moreover, experimental results show that neurotransmitter secretion rate exhibits fluctuations with time power law behaviour 17 and power laws are observed in fluctuations of extended excitable systems driven by stochastic fluctuations [18].

Here we present a model based on SOC ideas and taking into account synaptic plasticity in a neural network. With this model we analyse the time signal for electrical activity and compare the power spectra with EEG data. Plasticity is one of the most astonishing properties of the brain, occuring mostly during development and learning 19, 20, 21, and can be defined as the ability to modify the structural and functional properties of synapses. Modifications in the strength of synapses are thought to underly memory and learning. Among the postulated mechanisms of synaptic plasticity, the activity dependent Hebbian plasticity constitutes the most fully developed and influential model of how information is stored in neural circuits 22, 23, 24]. A large variety of models for brain activity has been proposed, based for instance on the convolution of oscillators [25] or stochastic waiting times 26]. They are essentially abstract representations on a mesoscopic scale, but none of them is based on the behaviour of a neural network itself. In order to get real insights on the relation between time series and the microscopic, i.e. cellular, interactions inside a neural network, it is necessary to identify the basic ingredients of the brain activity possibly responsible for characteristic scale-free behaviour observed through the spectrum power law.

In order to formulate a new model to study EEG signals, we introduce within a SOC approach the three 
most important ingredients for neuronal activity, namely threshold firing, neuron refractory period and activitydependent synaptic plasticity. We consider a simple square lattice of size $L \times L$ on which each site represents the cell body of a neuron, each bond a synapse. Therefore, on each site we have a potential $v_{i}$ and on each bond a conductance $g_{i j}$. Whenever at time $t$ the value of the potential at a site $i$ is above a certain threshold $v_{i} \geq v_{\max }$, approximately equal to $-55 \mathrm{mV}$ for the real brain, the neuron fires, i.e. generates an "action potential", distributing charges to its connected neighbours in proportion to the current flowing through each bond

$$
v_{j}(t+1)=v_{j}(t)+v_{i}(t) \frac{i_{i j}(t)}{\sum_{k} i_{i k}(t)}
$$

where $v_{j}(t)$ is the potential at time $t$ of site $j$, nearest neighbor of site $i, i_{i j}=g_{i j}\left(v_{i}-v_{j}\right)$ and the sum is extended to all nearest neighbors $k$ of site $i$ that are at a potential $v_{k}<v_{i}$. After firing a neuron is set to a zero resting potential. The conductances are initially all set equal to unity whereas the neuron potentials are uniformly distributed random numbers between $v_{\max }-2$ and $v_{\max }-1$. The potential is fixed to zero at top and bottom whereas periodic boundaries are imposed in the other direction.

The external stimulus is imposed at one input site in the centre of the lattice, and the electrical activity is monitored as function of time by measuring the total current flowing in the system. The firing rate of real neurons is limited by the refractory period, i.e. the brief period after the generation of an action potential during which a second action potential is difficult or impossible to elicit. The practical implication of refractory periods is that the action potential does not propagate back toward the initiation point and therefore is not allowed to reverberate between the cell body and the synapse. In our model, once a neuron fires, it remains quiescent for one time step and it is therefore unable to accept charge from firing neighbours. This ingredient indeed turns out to be crucial for a controlled functioning of our numerical model. In this way an avalanche of charges can propagate far from the input through the system.

As soon as a site is at or above threshold $v_{\max }$ at a given time $t$, it fires according to Eq. (1), then the conductance of all the bonds, connecting to active neurons and that have carried a current, is increased in the following way

$$
g_{i j}(t+1)=g_{i j}(t)+\delta g_{i j}(t)
$$

where $\delta g_{i j}(t)=k \alpha i_{i j}(t)$, with $\alpha$ being a dimensionless parameter and $k$ a unit constant bearing the dimension of an inverse potential. After applying Eq. (2) the time variable of our simulation is increased by one unit. Eq. (2) describes the mechanism of increase of synaptic strength, tuned by the parameter $\alpha$. This parameter

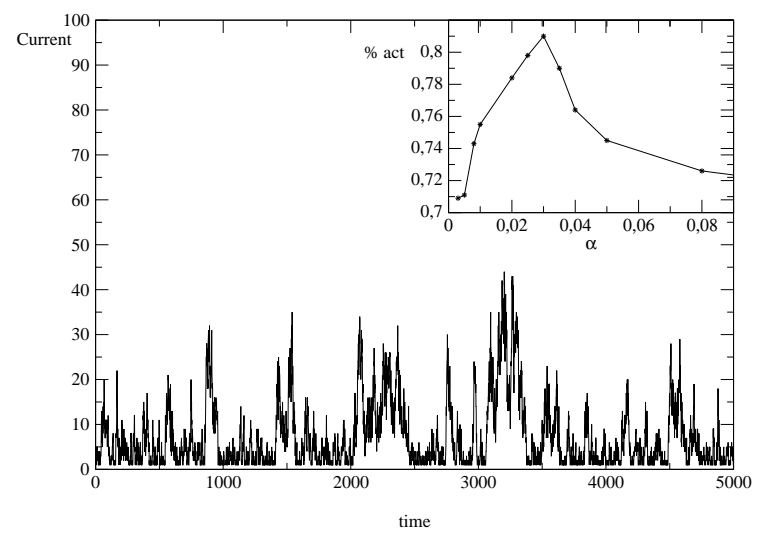

FIG. 1: Total current flowing in one square lattice configuration $\left(L=1000, \alpha=0.03, \sigma_{t}=0.0001, v_{\max }=6\right)$ as function of time in a sequence of several thousand stimuli. In the inset we show the asymptotic value of the percentage of active bonds as function of $\alpha$ for $L=100$. The value of the parameters is $\sigma_{t}=0.0001$ and $v_{\max }=6$.

then represents the ensemble of all possible physiological factors influencing synaptic plasticity, many of which are not yet fully understood.

Once an avalanche of firings comes to an end, the conductance of all the bonds with non-zero conductance is reduced by the average conductance increase per bond, $\Delta g=\sum_{i j, t} \delta g_{i j}(t) / N_{b}$, where $N_{b}$ is the number of bonds with non-zero conductance. The quantity $\Delta g$ depends on $\alpha$ and on the response of the brain to a given stimulus. In this way our electrical network "memorizes" the most used paths of discharge by increasing their conductance, whereas the less used synapses atrophy. Once the conductance of a bond is below an assigned small value $\sigma_{t}$, we remove it, i.e. set it equal to zero, which corresponds to what is known as pruning. This remodelling of synapses mimicks the fine tuning of wiring that occurs during "critical periods" in the developing brain, when neuronal activity can modify the synaptic circuitry, once the basic patterns of brain wiring are established [20]. These mechanisms correspond to a Hebbian form of activity dependent plasticity, where the conjunction of activity at the presynaptic and postsynaptic neuron modulates the efficiency of the synapse [24]. To insure the stable functioning of neural circuits, both strengthening and weakening rule of Hebbian synapses are necessary to avoid instabilities due to positive feedback 27]. However, differently from the well known Long Term Potentiation (LTP) and Long Term Depression (LTD) mechanisms, in our model the modulation of synaptic strength does not depend on the frequency of synapse activation [19, 28, 29].

The external driving mechanism to the system is imposed by setting the potential of the input site to the value $v_{\max }$, corresponding to one stimulus. This external stimulus is needed to keep functioning the system and 


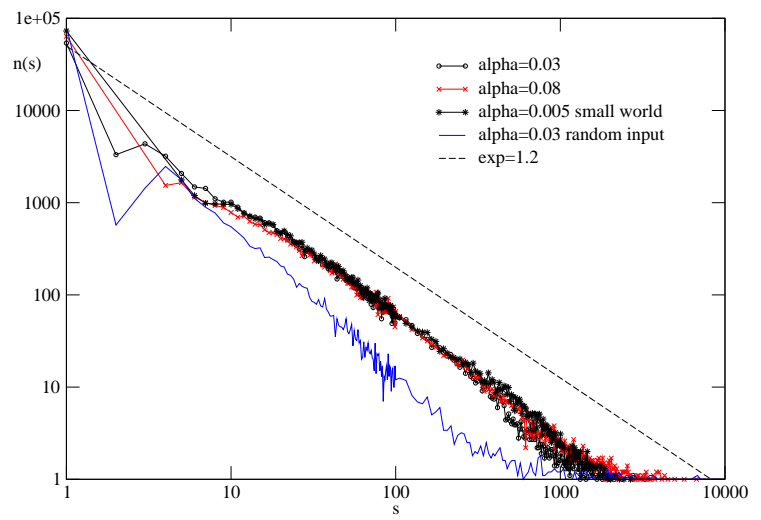

FIG. 2: (Color online) Log-log plot of the distribution of avalanche size $n(s)\left(L=1000, \alpha=0.03\right.$ and $0.08, N_{p}=10$, $\left.v_{\max }=6\right)$ for the square lattice (lines) and the small world lattice $\left(*, L=1000, \alpha=0.05, N_{p}=1000, v_{\max }=8\right)$ with $1 \%$ rewired bonds. The data are averaged over 10000 stimuli in 10 different configurations. The dashed line has a slope 1.2. For $\alpha=0.3$ and random input site the slope is 1.5.

therefore mimicks the living brain activity. We let the discharge evolve until no further firing occurs, then we apply the next stimulus. Fig.1 shows the electrical signal as function of time: the total current flowing in the system is recorded in time during a sequence of successive avalanches. Data show that discharges of all sizes are present in the brain response, as in self-organized criticality where the avalanche size distribution scales as a power law [5, 30] The strength of the parameter $\alpha$, controlling both the increase and decrease of synaptic strength, determines the plasticity dynamics in the network. For large values of $\alpha$ the system strengthens more intensively the synapses carrying current but also very rapidly prunes the less used connections, reaching after a short transient a plateau where it prunes very few bonds. On the contrary, for small values of $\alpha$ the system takes more time to initiate the pruning process and slowly reaches a plateau. The number of active (nonpruned) bonds asymptotically reaches its largest value at the value $\alpha=0.03$ (inset of Fig.1). This could be interpreted as an optimal value for the system with respect to plastic adaptation.

Since each avalanche may trigger the activity of a high number of neurons, large currents flow through the system, therefore after $N_{p}$ stimuli the network is no longer a simple square lattice due to pruning, but exhibits a ladder-like pattern with few lateral connections. This complex structure constitutes the first approximation to a trained brain, on which measurements are performed. These consist of a new sequence of stimuli at the input site, by setting the voltage at threshold, during which we measure the number of firing neurons as function of time. This quantity corresponds to the total current flowing in a discharge measured by the electromagnetic signal of the EEG. We have evaluated the size distribution of

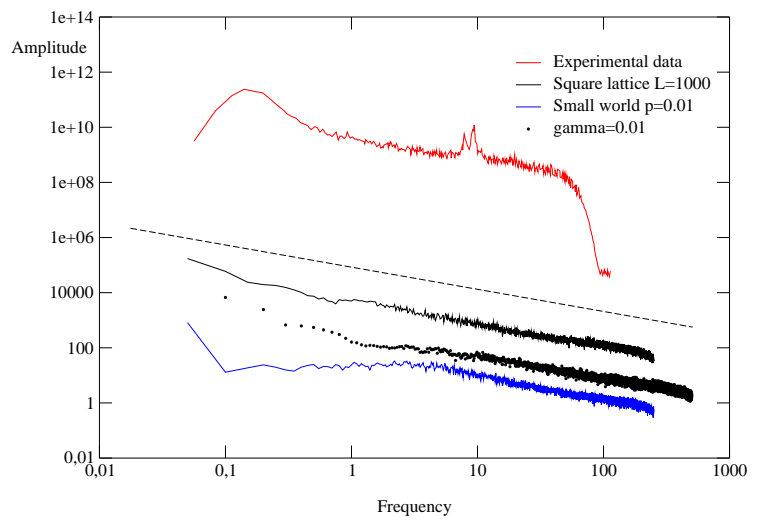

FIG. 3: (Color online) Power spectra for experimental data and numerical data $\left(L=1000, \alpha=0.03, N_{p}=10, v_{\max }=6\right)$ for the square lattice (middle line) and the small world lattice (bottom line, $L=1000, \alpha=0.05, N_{p}=1000, v_{\max }=8$ ) with $1 \%$ rewired bonds. Spectrum for the square lattice $\alpha=0.3$ and leaky neurons $(*, \gamma=0.01)$. The experimental data (top line) are from ref.[19] and frequency is in $\mathrm{Hz}$. The numerical data are averaged over 10000 stimuli in 10 different network configurations. The dashed line has a slope 0.8 .

neural avalanches, that is the total number of neurons involved in the propagation of each stimulus. This distribution exhibit power law behaviour, with an exponent equal to $1.2 \pm 0.1$, quite stable with respect to parameters (Fig.2). We have also simulated the brain dynamics on a square lattice with a small fraction of bonds, from 0 to $10 \%$, rewired to long range connections corresponding to a small world network [31, 32, 33], which more realistically reproduces the connections in the real brain. Fig.2 shows the size distribution scaling with an exponent $1.2 \pm 0.1$ for a system with $1 \%$ rewired bonds and a different set of parameters $\alpha, N_{p}, v_{\max }$. Conversely, for the input site chosen at random in the system, the scaling exponent changes and becomes $1.5 \pm 0.1$ (Fig.2).

In order to compare with medical data, we calculate the power spectrum of the resulting time series, i.e. the square of the amplitude of the Fourier transform as function of frequency. The average power spectrum as function of frequency is shown in a log-log-plot with the parameters $\alpha=0.03, N_{p}=10, \sigma_{t}=0.0001, v_{\max }=6$ and a lattice of size $L=1000$ (Fig. 3). We see that it exhibits a power law behaviour with the exponent $0.8 \pm 0.1$ over more than three orders of magnitude. This is precisely the same value for the exponent found generically on medical EEG power spectra [34, 35]. We also show in Fig. 3 the magnetoelectroencephalography (similar to EEG) obtained from channel 17 in the left hemisphere of a male subject, as measured in ref. [35], having the exponent 0.795 .

We have checked that the value of the exponent is stable against changes of the parameters $\alpha, v_{\max }, \sigma_{t}$, and $N_{p}$, and also for random initial bond conductances. Moreover, the scaling behaviour remains unchanged if 
the input site is placed at random in the system at each stimulus. For $\alpha=0$ the frequency range of validity of the power law decreases by more than an order of magnitude. Fig.3 also shows the power spectrum for a small world network with $1 \%$ rewired bonds and a different set of parameters $\alpha, N_{p}, v_{\max }$ : the spectrum has some deviations from the power law at small frequencies and tends to the same universal scaling behaviour at larger frequencies over two orders of magnitude. The same behaviour is found for a larger fraction of rewired bonds.

In real systems neurons have a leakage, namely the potential decays exponentially in time with a relaxation time $\tau$, i.e. $\frac{d v(t)}{d t}=-\gamma v(t)$, with $\gamma=1 / \tau$. Leakage has been considered in our model and the same scaling behaviour recovered (Fig.3). However for $\tau \leq 10$ (i.e. for stronger leaking), the low frequency part of the spectrum appears to be frequency independent and the scaling regime is recovered at high frequencies with an exponent in agreement with previous results.

In the mature living brain synapses can be excitatory or inhibitory, namely they set the potential of the postsynaptic membrane to a level closer or farther, respectively, to the firing threshold. We have introduced in our model this ingredient: each synapse is inhibitory with probability $p_{i n}$ and excitatory with probability $1-p_{i n}$. We have studied the power spectrum for a range of value of $p_{i n}$. For a density up to $10 \%$ of inhibitory synapses the same power law behaviour is recovered within error bars. For increasing density the scaling behaviour is progressively lost and the spectrum develops a complex multi-peak structure for $p_{i n}=0.5$. Furthermore, the size distribution exhibits an exponential behaviour even for very small densities of inhibitory synapses. These results suggest that the balance between excitatory and inhibitory synapses has a crucial role on the overall behaviour of the network, similarly to what can occur in some severe neurological and psychiatric disorders 36].

The stability of the spectrum exponent suggests that an universal scaling characterizes a large class of brain models and physiological signal spectra for brain controlled activities. Medical studies of EEG focus on subtle details of a power spectrum (e.g. shift in peaks) to discern between various pathologies. These detailed structures however live on a background power law spectrum that shows universally an exponent of about 0.8 , as measured for instance in refs. [34] and 35]. A similar exponent was also detected in the spectral analysis of the stride-to-stride fluctuations in the normal human gait which can directly be related to neurological activity [16]. Our simple model is based on SOC ideas: the threshold dynamics ensures time scale separation (slow external drive and fast internal relaxation). This dynamics leads to criticality and therefore power law behaviour [2]. However the new ingredients of the model, namely the plasticity of the synapses may be at the origin of the new observed exponent. This work may open new per- spectives to study pathological features of EEG spectra by including further realistic details into the neuron and synapsis behaviour.

Acknowledgements. We gratefully thank E. Novikov and collaborators for allowing us to use their experimental data. We also thank Salvatore Striano, MD, for discussions and Stefan Nielsen and Hansjörg Seybold for help. L.d.A. research is supported by EU Network MRTN-CT-2003-504712, MIURPRIN 2004, MIUR-FIRB 2001, CRdC-AMRA, INFM-PCI.

[1] P. Bak, How nature works. The science of self-organized criticality, Springer, New York, 1996.

[2] H.J. Jensen, Self-Organized Criticality, Cambridge University Press, Cambridge, 1998.

[3] S. Maslov, M. Paczuski, P. Bak, Phys. Rev. Lett. 73, 2162 (1994).

[4] J. Davidsen, M. Paczuski, Phys. Rev. E 66, 050101(R) (2002).

[5] J. M. Beggs, D. Plenz, J. Neurosci. 23, 11167 (2003).

[6] J. M. Beggs, D. Plenz, J. Neurosci. 24, 5216 (2004).

[7] P.Bak, C.Tang, J. Geophys. Res. 94, 15635 (1989).

[8] A. Sornette, D.Sornette, Europhys.Lett. 9, 197 (1989).

[9] P. Bak, K. Sneppen, Phys. Rev. Lett. 71, 4083 (1993);

[10] E.T. Lu, R.J. Hamilton, Astrophys. J. 380, L89 (1991)

[11] P. A. Politzer, Phys. Rev. Lett. 84, 1192 (2000).

[12] J. Faillettaz, F. Louchet, J.R. Grasso, Phys. Rev. Lett. 93, 208001 (2004).

[13] O. Peters, C. Hertlein and K. Christensen, Phys. Rev. Lett. 88, 018701 (2002)

[14] A. Gevins et al, Trends Neurosci. 18, 429 (1995).

[15] G. Buzsaki, A. Draguhn, Science 304, 1926 (2004).

[16] J. M. Hausdorff et al., Physica A. 302, 138 (2001).

[17] S.B. Lowen, S.S. Cash, M. Poo , M.C. Teich, J. Neuroscience, 17, 5666 (1997).

[18] D.R. Chialvo, G.A. Cecchi, M.O. Magnasco, Phys. Rev. E 61, 5654 (2000).

[19] T. D. Albraight et al, Neuron, Review supplement to vol.59 (February 2000).

[20] T. K. Hensch, Ann. Rev. Neurosci. 27, 549 (2004).

[21] L.F. Abbott, S.B. Nelson, Nature Neurosci. 3, 1178 (2000).

[22] D.O. Hebb, The organization of behaviour, New York: John Wiley, 1949.

[23] J.Z. Tsien, Curr. Opi.n Neurobiol. 10, 266 (2000); G.-Q. Bi, M.-M. Poo, Ann. Rev. Neurosci. 24, 139 (2001).

[24] S.J. Cooper, Neurosci. Biobehav. Rev. 28, 851 (2005).

[25] Y. Ashkenazy et al, Physica A. 316, 662 (2002).

[26] P. Ch. Ivanov et al, Europhys. Letters 43, 363 (1998).

[27] N.S. Desai, J. Physiol. Paris 97, 391 (2003).

[28] O. Paulsen, T. J. Sejnowski, Curr. Opin. Neurobiol. 10, 172 (2000).

[29] K. H. Braunewell, D.Manahan-Vaughan, Rev. Neurosci. 12, 121 (2001).

[30] The total current shows a pattern reminiscent of spontaneously occuring neuronal oscillations observed in developmental central nervous system networks, such as the so-called "giant depolarizing potentials", network-driven synaptic events observed in the immature hippocampus, when all circuits are excitatory. See Kasyanov A.M., Safi- 
ulina V.F., Voronin L.L., Cherubini E., Proc Natl Acad Sci USA, 1013967-3972 (2004).

[31] D. J. Watts, S. H. Strogatz, Nature 393, 440 (1998).

[32] L. F. Lago-Fernandez, R. Huerta,F. Corbacho,J.A. Siguenza, Phys. Rev. Lett. 84, 2758 (2000).

[33] O. Shefi, I. Golding, R. Segev, E. Ben-Jacob, A. Ayali, Physical Review E. 66, 021905 (2002).
[34] W. J. Freeman et al, J. Neurosci. Meth. 95, 111 (2000).

[35] E. Novikov, A. Novikov, D. Shannahoff-Khalsa, B. Schwartz, J. Wright, Physical Rev. E. 56, R2387 (1997).

[36] J.L. Rubenstein , M.M. Merzenich, Genes Brain Behav. 2, 255 (2003); E.M. Powell et al, J. Neurosci. 2, 622 (2003). 